\title{
An Improved Capture Data Processing Technology based on Human Eye'S Resolution
}

\author{
Xiangning Zhou, Zhiyong An \\ Key Laboratory of Intelligent Processing in Universities of Shandong, Shandong Institute of Business and Technology, Shandong \\ Province, China \\ 3977653@qq.com
}

\begin{abstract}
A large number of redundant data will be produced during the data capture processing. This paper based on the resolution of the human eye and the improved algorithm, uses the sensors as little as possible to capture the movement data in the skeletal animation technology. Then based on 3D virtual human model, more lifelike movement details will be generated. So that, we can capture the motion process of virtual people generally and take account of the virtual people movement details high fidelity.

Index Terms - Motion capture, Data processing, Human eye's resolution
\end{abstract}

\section{Introduction}

In commercial applications, more and more motion capture technology is used. Motion capture technology is through the system function module called sensor based on different measuring methods and analysis. We can get the relevant motion data of the participants from these sensors, and then generate the animation according to the data. The important reason why Motion capture technology is popular is that it can easily gain a lot of real data movement occurs, which reflect the real motion process [1]. And the data can sensitive the movement, which is important to the animation.

To capture more useful data, most motion capture system may improve the data capture frequency as much as possible. But a lot of redundant data is also captured simultaneously. During the virtual human motion control, approaching the excessive and inappropriate data would produce a certain degree of redundancy of distortion. Besides, too much useless data processing will reduce the efficiency of system. So, during rendering the animation according the captured data, some small movements will express a sudden distortion for the redundant data and the calculation error in the process. Despite the above these shortcomings, compared to the other technology motion capture technology can capture movement characteristics better.

\section{Model Representation}

As we all known, the human body is a very complex combination of system. It has more than 200 movements of freedom only from the angle. So the number of bone and joint is very great. How to convert the human skeletal to three dimension visual human real time in the computer is a difficult problem. And it is not necessary to use such an almost perfect virtual human model in actuality. And many a time, such an ideal model can be improve simplify to achieve a specific application requirements.

Considering the great sports mainly involves the joints about the human body, such as arms and legs, neck and the waist. So we must keep these joints and the freedom of the activities nodes. While, the other parts, such as eyes, mouth and toe can be approach as expanded nodes. Human skeleton model [2-3] is adopted and the bone and joint human body are showed as point and line.

For the model is showed by the tree structure, the virtual human model can be stored by the binary tree. To distinguish the left and right children, a special pointer to the parent node is added in each node in the virtual human model, which improves the efficiency of computing process.

\section{Data Processing}

\section{A. Human eye's resolution}

There are two black spots A and B in the screen. When the two spots getting more closer to a certain degree, the human eyes can not distinguish $\mathrm{A}$ and $\mathrm{B}$, that means the distinguishing ability of human eye on details of the scenery is limited and the ability is called human eyes resolution[4].

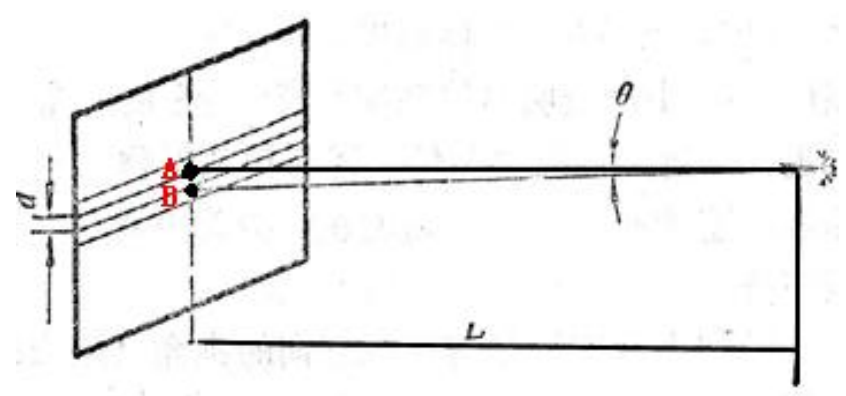

Fig.1 Human Eye's Resolution

From the fig.1, the distance between eyes and screen is $\mathrm{L}$. The adjacent distance between the two points which eyes can distingue is $d . \theta$ is visual angle. With the angle smaller, the resolution of the human eye is stronger. And human eye resolution can be defined by the reciprocal of the angle. By this reckoning, if the distances of the moving object moved less than $\mathrm{d}$, human eye can not notice.

$$
\operatorname{Tan} \theta=\mathrm{d} / \mathrm{L} \approx \theta, \theta^{\prime}=\theta \times 60 \times 360 / 2 \pi, \mathrm{d} \approx \theta^{\prime} \times \mathrm{L} / 3438 .
$$

\section{B. Evaluation function}

The biggest advantage of the Motion capture is it can capture the real data of human movement quickly and accurately. Because the generated movement simulated the main body movement in the reality, the effect is very realistic 
and complex. In order to reduce data redundancies and improve data access efficiency, a feasible way can be adopted. That is, all collected data information should be evaluated by an evaluation function in advance. If the influence to virtual people animation effect is in a negligible range, the data can be directly discarded. The evaluation function can be showed by a threshold function which is gotten by the human eyes resolution. And as we all known, the value of $\theta^{\prime}$ is between 1 to 1.5 minute of angle [4]. We can define the function as followed:

$$
\mathrm{d}\left(\mathrm{m}_{\mathrm{s}}\right)=\left\{\begin{array}{c}
0, m_{s}<d \\
1, m_{s}>=d
\end{array}\right.
$$

When the data from sensor corresponding to some joints is evaluated to eliminate in the pretreatment stage by evaluation function, that means, the displacement of the joints can not be distinguished by human eye, the data will be ignored and abandoned by system. And the system will think this joint does not move. At the end, animation effect is no changes. The exercise failed to be perceived will be accumulated to the next movement, until all the accumulated displacement can be perceived, these accumulated displacements will be compensated in the following movement process, which improves the reliability of the system to retentions of the movement.

A sufficient number of sensors are needed to install to capture enough data, if it is required to get more details about the movement process. From the experiments, we can get the conclusion that the number of sensors can be according to the detail requirement. On the one hand, it can reduce cost of the system; on the other hand, it can reduce the data processing of the system.

\section{Calculating displacement [5]}

For calculating displacement of virtual people's movement, the structure model of the $3 \mathrm{~d}$ virtual human which system used should be attend, the displacement of the joint in virtual people can be calculated by the following formula.

$$
\mathrm{M}=\mathrm{M}_{\mathrm{S}}+\mathrm{M}_{\mathrm{P}}
$$

$M$ is the displacement of the joint, $M_{S}$ is the displacement of the joint which the corresponding sensor measures. $M_{P}$ means the displacement of its parent joint. It should be attended that this is a recurrence formula, which adds all displacement of some joints' ancestors to its parents. So, the movement effect of every upper joint in the virtual human model will transfer to the lower level joints. If the calculated displacement is far greater than the distance which distinguished by the human eye's resolution, to show the movement smoothly, the intermediate frames will be insert between the start key frame and the end frame. Usually, the easy way is using linear insert algorithm.

For example, the coordinate of a virtual human joint in the virtual environment is $(\mathrm{x} 1, \mathrm{y} 1, \mathrm{z} 1)$. After $\mathrm{t}$, there is a movement displacement, dist and the captured coordinate by sensor is $(\mathrm{x} 2, \mathrm{y} 2, \mathrm{z} 2)$ at $\mathrm{t}+\Delta \mathrm{t}$. So, the position can be showed by the following formula.

$$
\mathrm{S}(\mathrm{t})=(\mathrm{x}(\mathrm{t}), \mathrm{y}(\mathrm{t}), \mathrm{z}(\mathrm{t}))
$$

The specific steps of the algorithm are as follows: Step 1: calculating the total displacement of the two key frames (Euclidean distance).

$$
\begin{aligned}
& \text { Dist } \\
& =|\mathrm{S}(\mathrm{t}+\Delta \mathrm{T})-\mathrm{S}(\mathrm{t})| \\
& =\sqrt{[x(t+\Delta t)-x(t)]^{2}+[y(t+\Delta t)-y(t)]^{2}+[z(t+\Delta t)-z(t)]^{2}}
\end{aligned}
$$

Step2: calculating he distance between the joint and the camera lens(c).

$\mathrm{L}$

$$
\begin{aligned}
& =|\mathrm{S}(\mathrm{t})-\mathrm{S}(\mathrm{c})| \\
& =\sqrt{[x(t)-x(c)]^{2}+[y(t)-y(c)]^{2}+[z(t)-z(c)]^{2}}
\end{aligned}
$$

Step 3: calculating the minimum distance $d$ and the number of the intermediate frame which should insert in the movement.

$$
\mathrm{N}=\mathrm{dist} / \mathrm{d}
$$

Step 4: for $\mathrm{I}=1$ to $\mathrm{N}$, calculate $\mathrm{S}(\mathrm{I})$

$$
\mathrm{S}(\mathrm{I})=\mathrm{S}(\mathrm{t})+\frac{I}{N}\left[\begin{array}{l}
x(t+\Delta t) \\
y(t+\Delta t) \\
z(t+\Delta t)
\end{array}\right]
$$

\section{Improved algorithm}

At present, the human eye can clearly see the area which the corresponding resolution is $2169 * 1213$.People can clearly see the field which the corresponding Angle is about 35 (horizontal) x20 (Vertical). According to the human eye's characteristics, the virtual camera view can be set to 35 (horizontal) x20 (Vertical). While, if the resolution of display and retina want to achieve consistent, the followed formula should be used:

$$
\mathrm{Cx}=\mathrm{cx}^{*} \mathrm{x} / 2169
$$

$$
\mathrm{Cy}=\mathrm{cy}^{*} \mathrm{y} / 1213
$$

$(\mathrm{x}, \mathrm{y})$ is the two-dimensional coordinates in the retina, $c x^{*} \mathrm{cy}$ is the resolution of the display device.(Cx, Cy)is the changed coordinated considering the device's resolution. In order to reduce the loss of the efficiency by the hardware equipment and as far as possible to ensure the accuracy of the motion, a intermediate frames can be inserted in reality. If the resolution of computer is $1280 * 800$, the Coefficient can be get ted from the formula:

$\mathrm{A}=\operatorname{Min}\{2169 / 1280,1213 / 800\}=\operatorname{Min}\{1.6945,1.5163\}=1.5163$

\section{Experimental Results}

Computer Configuration Item is: Intel Pentium Dual Core processor, $2 \mathrm{G}$ memory, $1 \mathrm{G}$ special Display Card, $\mathrm{VC}++$ 6.0, MSDirectXsDK, 3DsMAX.

This is an action that virtual human puts up his both hands, based on the above method and formulas, the frame screenshots are showed as followed. 

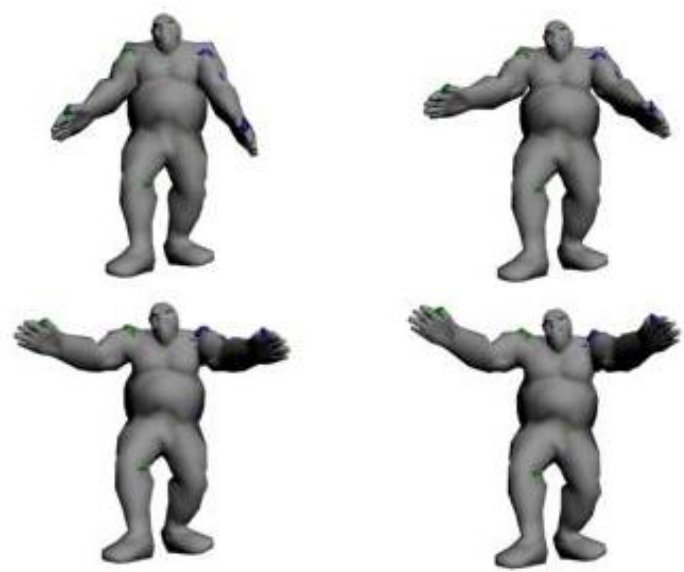

Fig. 2 Unimproved sequences of frames
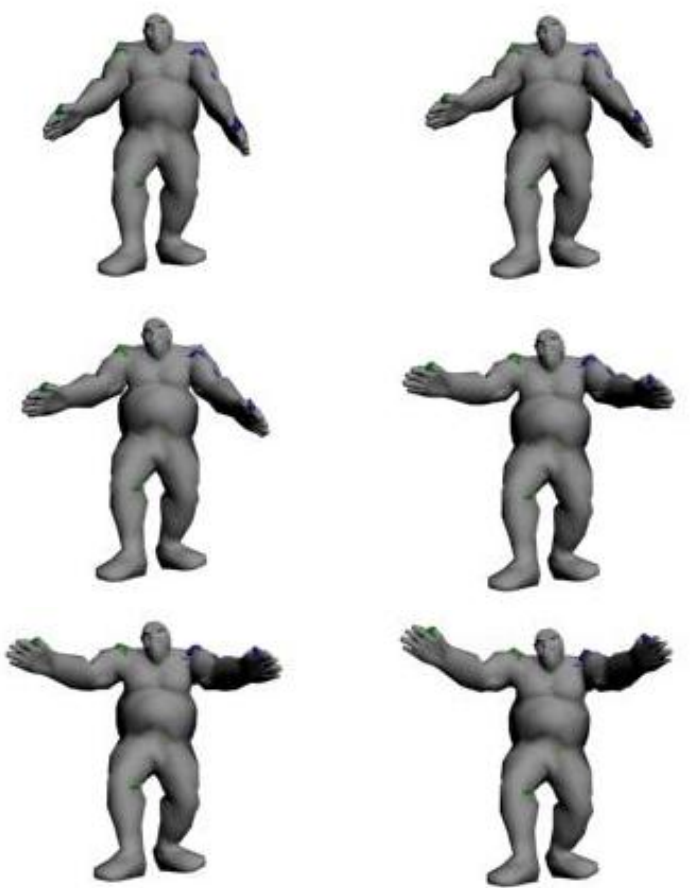

Fig.3 Improved sequences of frames
From the two figures, we can also find the difference of the movement. The improved algorithm can recognize the ignored movement details, which improves the capture's accuracy and recovers the movement process in a certain degree.

\section{References}

[1] Boxiang Xiao, "Data Processing, Retrieval and Reconstruction for Human Motion Capture", doctoral dissertation, 8-18(2009).

[2] Wang Zhaoqi, "Study on Synthesis of Virtual Human", Journal of the Graduate School of the Chinese Academy of Sciences, 17(2), 89-98(2000).

[3] C.Babski, D.Thalmann. "A Seamless Shape for HANIM Compliant Bodies". InProc. VRML9, 21-28(1999).

[4] Weizhen Wang," Research on a motion control technology of 3D virtual human based-on human eye's resolution", master paper, 22-23(2009).

[5] Xiangning Zhou, Capture data processing based on human eye's resolution. ICDIP2012, 8334-92. 